\title{
Unsupervised Machine Learning Neural Gas Algorithm for Accurate Evaluations of the Hessian Matrix in Molecular Dynamics
}

\author{
Michele Gandolfi and Michele Ceotto*
}

Cite This: J. Chem. Theory Comput. 2021, 17, 6733-6746

Read Online

ABSTRACT: The Hessian matrix of the potential energy of molecular systems is employed not only in geometry optimizations or high-order molecular dynamics integrators but also in many other molecular procedures, such as instantaneous normal mode analysis, force field construction, instanton calculations, and semiclassical initial value representation molecular dynamics, to name a few. Here, we present an algorithm for the calculation of the approximated Hessian in molecular dynamics. The algorithm belongs to the family of unsupervised machine learning methods, and it is based on the neural gas idea, where neurons are molecular configurations whose Hessians are adopted for groups of molecular dynamics configurations with similar geometries. The method is

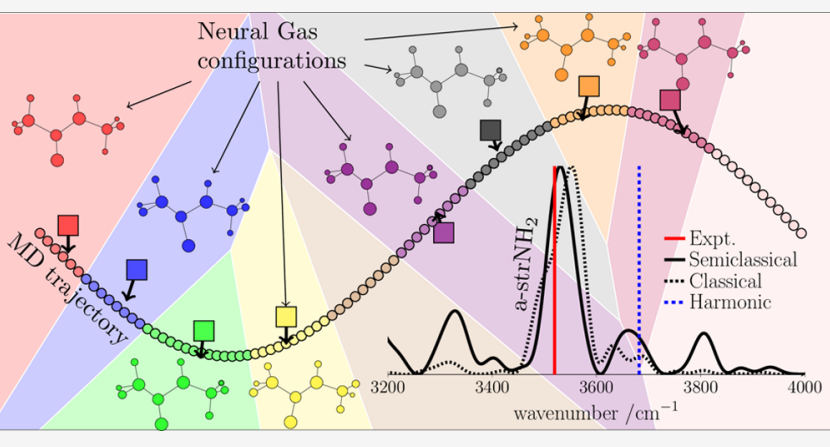
tested on several molecular systems of different dimensionalities both in terms of accuracy and computational time versus calculating the Hessian matrix at each time-step, that is, without any approximation, and other Hessian approximation schemes. Finally, the method is applied to the on-the-fly, full-dimensional simulation of a small synthetic peptide (the 46 atom $\mathrm{N}$-acetyl-L-phenylalaninylL-methionine amide) at the level of DFT-B3LYP-D/6-31G* theory, from which the semiclassical vibrational power spectrum is calculated.

\section{INTRODUCTION}

In standard molecular dynamics (MD) simulations, the atomic positions, velocities, and forces are evolved in time according to Hamilton's equations and calculated at each time-step. The physical interpretation of the atomistic details provided by dynamics simulations is very powerful and finds uncountable applications every day. However, if one looks for a deeper physical insight that requires information about the potential curvature, it becomes necessary to evaluate the Hessian (second-order derivatives of the potential energy) matrix at each time-step. Specifically, Hessians are employed for higher than second-order $\mathrm{MD}$ time-integrators, ${ }^{1-3}$ for geometry optimization calculations, ${ }^{4,5}$ for instantaneous normal mode analysis, ${ }^{6,7}$ for accurate force field constructions, ${ }^{8}$ for semiclassical dynamics, ${ }^{9}$ and other applications, such as reaction rate constants with the instanton method. ${ }^{10,11}$ While integration of Hamilton's equations of motion is doable for any number of degrees of freedom, assuming that the interacting potential is readily available as well as that there is suitable computational power, computing properties that depend on the second or even higher coordinate derivatives of the potential is a challenging task since these calculations usually scale polynomially with the system size. The task may become prohibitive in $a b$ initio $\mathrm{MD}^{12}$ where the potential and its derivatives are evaluated on-the-fly, that is, by solving the electronic structure problem and using the Hellman-Feynman theorem, or by the finite difference formula using the forces or the potential. To address this issue, a number of approximate methods have been introduced. ${ }^{13-16}$ Usually, these are of the type of updating schemes, where the Hessian is approximated in a step-wise fashion using the latest information available. ${ }^{17}$ These updating schemes were originally developed for optimization $^{17-21}$ (see also references therein) but have much evolved and improved since then. Later, they have been employed in various algorithms for direct dynamics simulations. ${ }^{1-3}$ For example, the Broyden method is based on a first-order Taylor expansion, which is equivalent to the quasiNewton methods employed in optimization processes. However, in $a b$ initio MD, a higher accuracy is desirable as it has been shown how a highly accurate Hessian approximation can attain high simulation quality. ${ }^{13}$ More recently, Denzel and Kästner ${ }^{22}$ followed another route to face the problem, which is to use the Gaussian process regression method ${ }^{23-25}$ to generate a local fit of the potential surface (GPR-PES), possibly using Hessians as fitting variables. Then, the GPR-PES can be differentiated analytically as many times as required,

Received: July 14, 2021

Published: October 27, 2021 


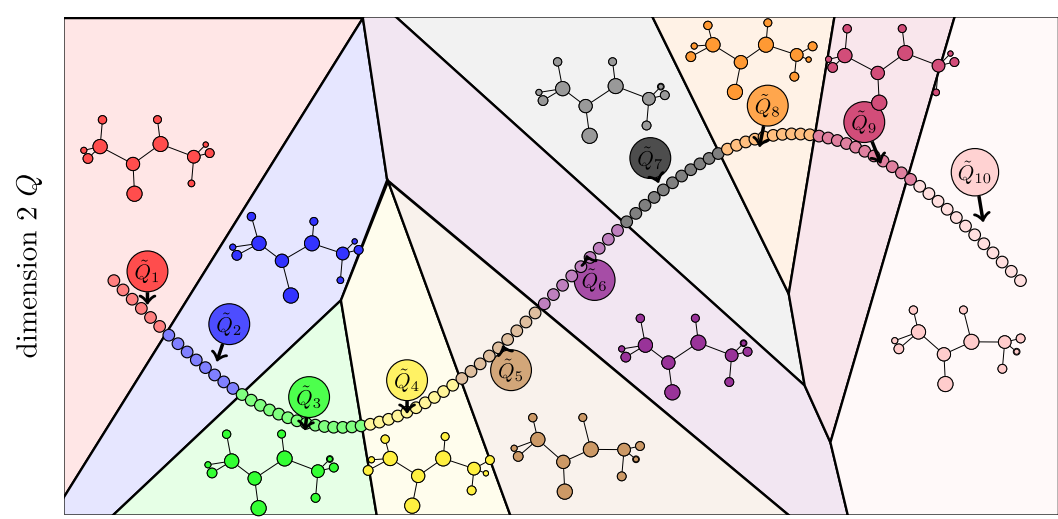

dimension $1 Q$

Figure 1. Pictorial representation of neuron adaptation for a MD trajectory manifold in a convenient 2D plane. The trajectory configurations are represented by the collection of small circles, and the neuron positions are the larger circles labeled by $\tilde{Q}_{i}$ for the $i$-th neuron. The arrows at the neuron circles represent the updating coordinate direction. The domains of each neuron are bordered by solid lines and identified by different colors. All geometries within the same domain share the same Hessian matrix, which is the one calculated at the neuron location.

providing accurate Hessian matrices. The method has been successfully employed in various applications ranging from accurate instanton calculations ${ }^{26}$ to the modeling of molecular, amorphous materials and surfaces (see ref 25 and references therein), to mention some. However, the GPR-PES method (including Hessian estimation) was intended to give an accurate description of only a local region of the PES. Hence, it is unsuited for extensive MD simulations. Furthermore, the GPR fitting time and memory usage scale unfavorably with the system size, and the method is not recommended for systems with more than 100 degrees of freedom, as the authors pointed out. ${ }^{22}$

In this paper, we take a different strategy from those described above for the Hessian approximation. The idea is to assign the same Hessian matrix to a group of MD trajectory configurations that are characterized by similar geometric properties. Since the Hessian ultimately depends on the potential energy surface (PES), we think that the collection of molecular coordinates is an appropriate set of variables to combine a group of configurations, given that the Hessian is uniquely defined for each set of atomic coordinates. Specifically, we employ the unsupervised machine learning algorithm "neural gas" to clusterize similar coordinates. The neural gas (NGas) algorithm was originally proposed as a selforganizing-map or a self-organizing-network by Martinetz and Schulten $^{27}$ in 1991, with the objective of learning the dimensions and topology of a generic manifold of simple geometrical shapes and complicated time series. ${ }^{28}$ The algorithm devised by Martinetz, Berkovich, and Schulten features a number of landmark coordinates called neurons that are initialized nearby the objective manifold either randomly or according to some rule. Then, the neurons gradually adapt and connect to best represent the manifold shape, thus arranging in the manifold as an approximate time series. For the adaptation to be effective, the algorithm iteratively drags the neurons closer to the manifold in a way to minimize a given error function, which can be, for example, the sum of the Euclidean distances of each neuron from the collection of events in the time series. As a final result, the manifold is divided into optimal domains, the Voronoi cells, one for each reference neuron.

An intermediate step of the algorithm is pictorially represented in Figure 1, where the time series of configurations is reported as a line of small circles and the neuron locations as larger circles. The molecular geometry of each neuron is pictorially represented. The collection of the trajectory configurations that are related to each neuron are distinguished by a color code, and the neuron domains are bordered by continuous black lines. In a few words, molecular geometries of the same color share the same Hessian, which is the one calculated at the corresponding neuron geometry. The time series of configurations which form the manifold can be generated by many trajectories as well. The procedure avoids any redundancy that a multiple trajectory time series may generate when trajectories have crossing paths. This algorithm will take advantage of the fact that bound system trajectories are subject to visit the same phase space neighborhood several times during the dynamics, a point which is missed by the Hessian update schemes (vide infra). ${ }^{16}$ As a matter of fact, neurons undergo a competitive behavior in getting closer to larger portions of manifold conformations, and more probable phase space regions will exhibit higher densities of neurons. Also, we expect that, as shown in Figure 1, for a curved trajectory, the optimal neuron location would be nearby the center of curvature, which is equally representative of the curvature geometries. Instead, when the trajectory lies on a straight line, the optimal neuron location would be on top of the trajectory. A modified version of the NGas algorithm was introduced in 1994 by Fritzke. ${ }^{29}$ In this version, it is not required to specify the number of input neurons, with new neurons being added as the optimization proceeds. Later on, many groups provided further advances and optimizations on top of the original version to enforce topology preservation ${ }^{30,31}$ and allow for a better scaling and optimal growth with increasing amount of data. ${ }^{31-33}$

The use of supervised and unsupervised machine learning algorithms for molecular modeling has a long history in the field of quantitative structure-activity relationships (QSAR). ${ }^{34}$ Different kinds of molecular descriptors ${ }^{35}$ are employed to predict a plethora of properties, especially in the field of medicinal chemistry ${ }^{36}$ and drug discovery. ${ }^{37,38}$ In recent years, supervised algorithms have been recognized as powerful tools in the formal field of theoretical physical chemistry (see ref 39 for an insightful perspective), also for MD simulations. ${ }^{40-42}$ In addition, unsupervised algorithms have found successful 
applications in (al)chemical space exploration and chemical design. $^{43-45}$

In this paper, we show that the unsupervised machine learning algorithm "neural gas" can optimally compress the information contained in simple molecular geometries along a MD simulation, and we use the compressed information to approximate the Hessian matrix. More specifically, in this work, we develop and test a NGas method for Hessian approximation with applications to the computation of vibrational spectra using the semiclassical initial value representation (SCIVR) method ${ }^{9,46}$ with the divide-andconquer technique (DC SCIVR) implementation developed by our group. ${ }^{47}$ In fact, the bottleneck of SCIVR dynamics is the computation of the Hessian matrix along the trajectories.

The paper is organized as follows: in the Methods section, we present in detail the NGas method implementation for the Hessian approximation, after recalling other two methods for Hessian approximation that we will compare with. Then we briefly recall the approach we use for the computation of vibrational power spectra in the semiclassical approximation, and eventually in the Results section, we apply the method to several molecular systems of growing dimension up to a small synthetic peptide. We conclude the paper with a summary and discussion of our findings.

\section{METHODS}

Compact Finite Difference Methods. In previous publications, ${ }^{14,15}$ Ceotto, Zhuang, and Hase have presented and showed how to employ a Hessian updating scheme based on a compact finite difference (CFD) strategy for MD simulations. ${ }^{48-51}$ The CFD approach allows one to obtain a high-order finite difference approximation of function differentiations without incurring a large stencil. This goal is achieved by including differentiated terms at more locations within a "compact" stencil. In this updating scheme, the Hessian is estimated by extrapolation. For example, if the MD geometry $X_{i}=\left(x_{i, 1}, x_{i, 2}, \ldots, x_{i, n}\right)$ of $n$ scalar entries is followed by $X_{i^{\prime}}$ at a later time, the updating scheme $H\left(X_{i^{\prime}}\right)=H\left(X_{i}\right)+\Delta H$ allows one to estimate the Hessian for the later geometry $H\left(X_{i^{\prime}}\right)$ once $\Delta H$ is estimated. Bofill ${ }^{21}$ proposed the following update recipe

$$
\begin{aligned}
\Delta H= & (1-\lambda) \frac{R \otimes R^{\mathrm{T}}}{R^{\mathrm{T}} \cdot \Delta X}+\lambda\left(\frac{\Delta X \otimes R^{\mathrm{T}}+R \otimes \Delta X^{\mathrm{T}}}{\|\Delta X\|^{2}}\right. \\
& \left.-\frac{R^{\mathrm{T}} \cdot \Delta X}{\|\Delta X\|^{4}} \Delta X \otimes \Delta X^{\mathrm{T}}\right)
\end{aligned}
$$

where $\lambda$ is a parameter allowed to vary, $\Delta X=X_{i^{\prime}}-X_{i}$

$$
R=2\left[G\left(X_{i^{\prime}}\right)-G\left(X_{i}\right)-H\left(X_{i}\right) \cdot\left(X_{i^{\prime}}-X_{i}\right)\right]
$$

$G(X)$ is the gradient and $\otimes$ and $\cdot$ are the symbols for outer and inner products of vectors. ${ }^{13}$ When $\lambda=0$, the CFD-symmetric rank-one scheme ${ }^{19}$ is derived, while the CFD-power symmetric Broyden scheme is obtained with $\lambda=1$, and the CFD-Bofill family schems is represented by the set of linear combinations between the two. Bofill ${ }^{21}$ suggested the following practical value for $\lambda$

$$
\lambda=1-\frac{\left(R^{\mathrm{T}} \cdot \Delta X\right)^{2}}{\|R\|^{2}\|\Delta X\|^{2}}
$$

which avoids the singularity division by near-zero when $R$ is almost orthonormal to $\Delta X$ in the first term of eq 1 . This choice was reported to be quite an accurate Hessian approximation. ${ }^{13}$ We provide both our implementation and the pseudocode in the Supporting Information.

Dynamical Hessian Database Methods. An alternative strategy proposed by our group is to create a dynamical database of Hessians (DBH) and related geometries. ${ }^{16}$ The idea is to approximate $H\left(X_{i^{\prime}}\right) \approx H\left(X_{i}\right)$ at the MD configuration $X_{i^{\prime}}$, whenever $X_{i^{\prime}}$ is a geometry close enough to $X_{i}$, that is, a geometry which has already been saved in a database. Two molecular configurations $X_{i}=\left(x_{i, 1}, x_{i, 2}, \ldots, x_{i, n}\right)$ and $X_{i^{\prime}}$ are considered close enough when

$$
\sqrt{\frac{\sum_{k=1}^{n}\left(x_{i, k}-x_{i^{\prime}, k}\right)^{2}}{n}}<\rho
$$

or

$$
\left|x_{i, k}-x_{i^{\prime}, k}\right|<\rho, \quad \forall k=1, \ldots, n
$$

that is, their distance is smaller than a given threshold $\rho$. Equation 4 is less strict than eq 5 , and hence we adopt the latter (eq 5) for the simulations presented below. To avoid database search latency time, $\left|x_{i, k}-x_{i^{\prime}, k}\right|$ can be evaluated mode after mode only for those geometries satisfying the threshold condition. If more than one geometry satisfies eq 5 , then the Hessian is approximated by the one associated with the geometry with the smallest difference in eq 5 . The database may be updated step by step during the MD simulation, or it may be created once from a given trajectory. In both cases, only the Hessians for those geometries which do not satisfy the requirement in eq 5 are computed, and the corresponding entry is saved in the database. This method has been extensively tested. ${ }^{16}$ The method has allowed for semiclassical simulation of systems where the computational time would have been otherwise too demanding. More details can be found in ref 16 . We provide both our implementation and the pseudocode in the Supporting Information of this paper.

NGas Algorithm for Hessian Approximation. We borrow from the DBH method the idea that close enough molecular configurations have similar Hessians, but we add the feature that the algorithm is allowed to look for optimal configurations even outside the trajectory pathway. As described above, the idea of the NGas method is to approximate a given set of elements with few representative ones, called neurons. In the case of the set of Hessian matrices along a classical trajectory, a NGas algorithm would find few geometries whose Hessians can be employed to approximate the Hessian matrix at every configuration along the trajectory.

Notice that all methods shown in this paper are agnostic with respect to the coordinate system and units. In our case, we usually perform MD either in Cartesian or normal mode coordinates. However, we ultimately employ mass-scaled normal mode coordinates for our spectra calculations. To locate the neurons and proceed with the NGas optimization process, we first scale the whole trajectory set of coordinates to fit a cubic box with edge 1 . In other words, we map each massscaled normal mode coordinate component $q_{j}(t)$ according to the equation

$$
Q_{j}(t)=\frac{q_{j}(t)-m_{j}}{M_{j}-m_{j}}
$$

where $m_{j}=\min q_{j}(t)$ is the minimum value in the time series of the $j$-th component, $M_{j}=\max q_{j}(t)$, and the new coordinates 
$\mathbf{Q}(t)$ are the scaled coordinates with values between 0 and 1 . Once the number of neurons, that is, the number of most representative geometries, is chosen, we evenly sample the initial guess $s$ for the neuron positions directly from the set $\mathbf{Q}(t)$ of the trajectory geometries, that is, initially the set $\{\tilde{\mathbf{Q}}\} \subset$ $\{\mathbf{Q}(\mathrm{t})\}$. In practice, a fixed number of neurons are initialized on top of the trajectory configurations in normal mode coordinates and distributed at fixed time intervals. In their first presentation of the NGas algorithm, Martinez and Schulten ${ }^{27}$ suggested that at each epoch $\tau$, all trajectory geometries $\mathbf{Q}_{i}$ are sampled in a random order from the set of trajectory configurations $\{\mathbf{Q}(t)\}$ (without repetition). Every time a configuration $\mathbf{Q}_{i}$ is sampled, each $j$-th neuron $\tilde{\mathbf{Q}}_{j}$ is updated according to following rule

$$
\tilde{\mathbf{Q}}_{j}=\tilde{\mathbf{Q}}_{j}+\alpha(\tau) \mathrm{e}^{-K_{i j} / \lambda(\tau)}\left(\tilde{\mathbf{Q}}_{j}-\mathbf{Q}_{i}\right)
$$

where $K_{i j}$ is an integer number that ranks the distance between the trajectory scaled coordinate geometry $\mathbf{Q}_{i}$ and the neuron $\tilde{\mathbf{Q}}_{\text {. }}$ Specifically, $K_{i j}$ is equal to 0 for the nearest trajectory geometry and to $(n-1)$ for the furthest one. In eq 7, $\alpha(\tau)$ and $\lambda(\tau)$ are parameters which are modeled to decrease during the optimization process. These parameters change for each epochal iteration, and they tune the NGas adaptability, that is, its ability to expand and how fast this expansion is performed. More specifically, $\lambda$ tunes the number of neighbor coordinates that can significantly interact with each neuron, while $\alpha$ tunes the adaptability of the NGas. In other words, the larger the $\lambda$, the greater the number of trajectory geometries that significantly contribute to the updating scheme in eq 7 , while $\alpha$ tunes how large the response of $\tilde{\mathbf{Q}}$ is and after how many iterations it is still responsive and learning. $\alpha$ and $\lambda$ are updated at each epoch with the same rule ${ }^{28}$

$$
g(\tau)=g_{\text {init }}\left(\frac{g_{\text {final }}}{g_{\text {init }}}\right)^{\tau / \tau_{\max }}
$$

with $g_{\text {init }}$ and $g_{\text {final }}$ being parameters. Reasonable choices for these parameters are $\alpha_{\text {init }}=0.3, \alpha_{\text {final }}=0.05, \lambda_{\text {init }}=30$, and $\lambda_{\text {final }}$ $=0.01$, independently of the simulated system. ${ }^{28}$

The updating formula in eq 7 can also be written using the $\mathcal{M}_{i j}$ operator formalism that we introduce here

$$
\begin{aligned}
\mathcal{M}_{i j} \tilde{\mathbf{Q}}_{j} & =\tilde{\mathbf{Q}}_{j}\left(1+\alpha(\tau) \mathrm{e}^{-K_{i j} / \lambda(\tau)}\right)-\alpha(\tau) \mathrm{e}^{-K_{i j} / \lambda(\tau)} \mathbf{Q}_{i} \\
& =\tilde{\mathbf{Q}}_{j}\left(1+A_{i j}(\tau)\right)-A_{i j}(\tau) \mathbf{Q}_{i}
\end{aligned}
$$

where $\alpha(\tau)$ and $\lambda(\tau)$ have been grouped into one parameter $A_{i j}(\tau)=\alpha(\tau) \mathrm{e}^{-K_{i j} / \lambda(\tau)}$, which depends on $\alpha, \lambda$, and $K_{i j} . A_{i j}(\tau)$ has the form of a Boltzmann factor with temperature $\lambda(\tau)$, and it is interpreted as a kind of neuron "influence probability." As the NGas training goes on, $\lambda(\tau)$ (the analogous of temperature) decreases and the gas freezes nearby the trajectory. Assuming that we know beforehand all $K_{i j}$ coefficients for the motion of the neuron $\tilde{\mathbf{Q}}_{j}$ (in general we do not), by applying the $\mathcal{M}_{i j}$ operator of eq 9 for $N_{\text {steps }}$ time-steps, that is, for the whole set of trajectory points $\{\mathbf{Q}(t)\}$, in a random order and without repetition, one gets

$$
\begin{gathered}
\mathcal{M}_{p(i, 1), j} \ldots \mathcal{M}_{p(i, n), j} \tilde{\mathbf{Q}}_{j}=\tilde{\mathbf{Q}}_{j} \prod_{u=1}^{N_{\text {steps }}}\left(1+A_{p(i, u) j}\right) \\
-\sum_{u=1}^{N_{\text {steps }}} \mathbf{Q}_{p(i, u)} A_{p(i, u) j} \prod_{v=u+1}^{N_{\text {steps }}}\left(1+A_{p(i, v) j}\right) \\
=\tilde{\mathbf{Q}}_{j} \prod_{u=1}^{N_{\text {steps }}} B_{i u j}(\tau) \\
-\sum_{u=1}^{N_{\text {steps }}} \mathbf{Q}_{p(i, u)}\left(B_{i u j}(\tau)-1\right) \prod_{v=u+1}^{N_{\text {steps }}} B_{i v j}(\tau)
\end{gathered}
$$

where $p(i, u)$ is the $u$-th element of a random permutation of the index $i$ over the list of the first $N_{\text {steps }}$ natural numbers, and we wrote the matrix with permuted indices as a three indice tensor: $B_{i u j}(\tau)=1+A_{p(i, u) j}(\tau)$. Equation 10 accounts for the core functionality of the NGas algorithm. In eq 10, the first term on the right-hand side does not depend on the trajectory position but only on the trajectory distance ranking from the $j$ th neuron $\tilde{\mathbf{Q}}_{\text {, in }}$ the form of the $K_{i j}$ coefficients. Instead, the second term in eq 10 depends on the trajectory position $\mathbf{Q}_{p(i, u)}$ both explicitly and implicitly through $K_{i j}$. Eventually, when $\lambda$ tends to 0 as described above, also $A_{i j}$ goes to 0 and the sums and products in eq 10 converges to a final neuron position with less than $N_{\text {steps }}$ terms. Within the analogy of the NGas, we would say that in the case when the gas is cold, it is influenced only by the local manifold (nearby trajectory points) rather than by the whole environment (the entire trajectory points), even if all trajectory configurations are summed by the index $u$ in eq 10 . However, even if eq 10 has been introduced to better understand the physics of the NGas iterations, eq 7 is employed in practice. According to these equations, the NGas process is a competitive type of learning since neurons compete to be nearest as possible to the trajectory geometries. This competitiveness is encoded in the parameters $K_{i j}$, which may change every time a neuron is moved and make it impossible to use eq 10 straightforwardly.

Once the gas is frost, we perform further optimization of each neuron position $\tilde{\mathbf{Q}}_{\mathbf{j}}$. First, we consider that for each trajectory point $\mathbf{Q}_{i}$, there is only one nearest neuron position $\tilde{\mathbf{Q}}$. Then, we collect all these points into a set $\{\mathbf{Q}(t)\}_{j}$ which is the collection of trajectory segments nearest to the $j$-th neuron, and there will be as many sets of this type as the number of neurons. Eventually, we can estimate the error $E\left(\tilde{\mathbf{Q}}_{i}\right)$ to consider the neuron $\tilde{\mathbf{Q}}_{\dot{j}}$ at the place of the trajectory segments $\mathbf{Q}(t)$ as the line integral

$$
E\left(\tilde{\mathbf{Q}}_{j}\right)=\frac{1}{V} \int_{\{Q(t)\}_{j}}\left(\tilde{\mathbf{Q}}_{j}-\mathbf{Q}(t)\right)^{2} \mathrm{~d} s
$$

where $V=\int_{\{Q(t)\}_{j}} \mathrm{~d} s$ is a normalization constant and $\mathrm{d} s$ is the integration line segment. Now, we can locate $\tilde{\mathbf{Q}}_{\text {such that }}$ $E\left(\tilde{\mathbf{Q}}_{j}\right)$ is minimal. The first-order derivative of $E\left(\tilde{\boldsymbol{Q}}_{j}\right)$ with respect to each $\tilde{\mathbf{Q}}_{j}$ is

$$
\begin{aligned}
\nabla_{\tilde{\mathbf{Q}}_{j}} E\left(\tilde{\mathbf{Q}}_{j}\right) & =\frac{2}{V} \int_{\{\mathbf{Q}(t)\}_{j}}\left(\tilde{\mathbf{Q}}_{j}-\mathbf{Q}(t)\right) \mathrm{d} s \\
& =2\left(\tilde{\mathbf{Q}}_{j}-\frac{1}{V} \int_{\{\mathbf{Q}(t)\}_{j}} \mathbf{Q}(t) \mathrm{d} s\right) \\
& =2\left(\tilde{\mathbf{Q}}_{j}-\langle\mathbf{Q}(t)\rangle_{j}\right)
\end{aligned}
$$




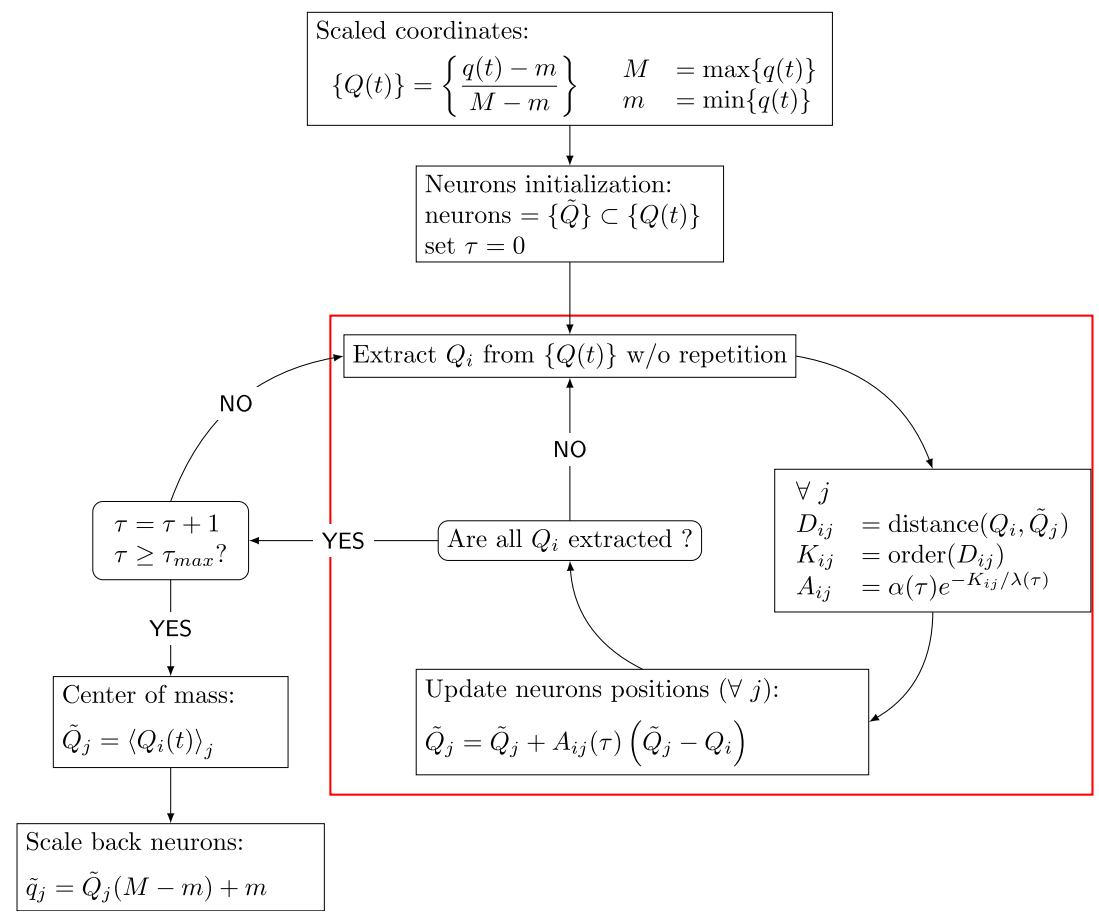

Figure 2. Flow diagram of our NGas implementation. Neurons are sampled from the scaled coordinates and iteratively optimized according to the cyclic part of the diagram. Every time a coordinate $\mathbf{Q}_{i}$ is sampled, one needs to compute its distance from every neuron to determine the ordering (encoded in the $\mathbf{K}$ matrix). Once the training is done, the neurons are scaled back to their original normal mode or Cartesian form. A red rectangular frame delimits the core part of the NGas algorithm, where neurons are updated in competition with one another to get closer to the trajectory.

Equation 12 is equal to 0 when $\tilde{\mathbf{Q}}_{\mathbf{j}}$ is equal to the "center of mass" $\langle\mathbf{Q}(t)\rangle_{j}$ of the trajectory segments $\{\mathbf{Q}(t)\}_{j}$. Hence, we implemented into the algorithm this further optimization step such that each neuron is eventually placed at the center of mass with respect to the trajectory points associated with that neuron.

Figure 2 reports the flow diagram of the algorithm described above, with the core part of the algorithm enclosed by the red rectangular frame. Apart from the scaling and the final optimization steps, the algorithm can be traced back to the first version by Martinetz and Schulten. ${ }^{27}$ At each epoch, all trajectory coordinates $\mathbf{Q}_{i}$ enter in a random order the NGas optimization cycle, where the distance $D_{i j}$ from its nearest neuron $\tilde{\mathbf{Q}}_{j}$ is evaluated together with the order coefficient $K_{i j}$. The epoch step is completed only after all trajectory points have been considered and the related neuron updated according to eq 7. For the following epoch, $A_{i j}(\tau)$ is updated and so on. At the end of the epoch evolution, each $j$-th neuron coordinate is placed at the center of mass of the collection of trajectory points that are nearest to that neuron $\{\mathbf{Q}(t)\}_{j}$. The new location $\tilde{\mathbf{Q}}_{\dot{j}}$ is then transformed back into the original trajectory coordinate system of reference, $\tilde{\mathbf{q}}_{j}$, that can be either Cartesian or normal mode ones. Recently, an algorithm ${ }^{52}$ that uses the idea of dividing the configuration space in Voronoi cells (as in the NGas method) has been proposed. The algorithm creates an on-the-fly updated mesh to approximate the potential energy from previous potential and potential gradient evaluations.

We evaluate the quality of the approximation as the mean absolute error (MAE) of the Cartesian Hessian matrix elements

$$
\sigma_{\text {Hess }}=\frac{1}{N_{\text {steps }} N_{\text {cart }}^{2}} \sum_{k}^{N_{\text {steps }}} \sum_{i}^{N_{\text {cart }}} \sum_{j}^{N_{\text {cart }}}\left|H_{i j}(k)-H_{i j}^{\text {approx }}(k)\right|
$$

where $N_{\text {cart }}$ is the number of Cartesian coordinates, $N_{\text {steps }}$ is the number of $\mathrm{MD}$ time-steps, $H_{i j}(k)$ is the entry of the exact Hessian matrix, and $H_{i j}^{\text {approx }}(k)$ is the approximated one, both at step $k$. We provide both our implementation and the pseudocode in the Supporting Information.

\section{SEMICLASSICAL INITIAL VALUE REPRESENTATION VIBRATIONAL SPECTROSCOPY}

In this paper, we will employ the NGas approximation described above for the calculation of Hessians in semiclassical dynamics for spectroscopy calculations. The semiclassical power spectrum $I(E)$ of a system of Hamiltonian $\hat{H}$ can be written as the Fourier-transformed wavepacket survival amplitude (in atomic units) ${ }^{53-55}$

$$
I(E)=\frac{1}{2 \pi} \int_{-\infty}^{+\infty} \mathrm{e}^{\mathrm{i} E t}\langle\chi \mid \chi(t)\rangle \mathrm{d} t
$$

where $|\chi(t)\rangle=\mathrm{e}^{-\mathrm{i} \hat{H} t}|\chi\rangle$ is the quantum time-evolution of the arbitrary reference state $|\chi\rangle$. The power spectrum provides the collections of all vibrational eigenvalues on an absolute scale. We calculate eq 14 using the time-averaging semiclassical initial value representation (TA SCIVR) method, ${ }^{9,56-64}$ where a time-averaging filter is applied to the semiclassical HellerHerman-Kluk-Kay (HHKK) propagator. ${ }^{65-77}$ The TA SCIVR expression of eq 14 for a system characterized by $N_{\text {vib }}$ degrees of freedom is 


$$
\begin{aligned}
& I(E)=\left(\frac{1}{2 \pi}\right)^{N_{\text {vib }}} \iint \mathrm{d} \mathbf{p}_{0} \mathrm{~d} \mathbf{q}_{0} \frac{1}{2 \pi T} \\
& \left|\int_{0}^{T} \mathrm{~d} t\left\langle\chi \mid \mathbf{p}_{t} \mathbf{q}_{t}\right\rangle \mathrm{e}^{i\left(S_{t}\left(\mathbf{p}_{0}, \mathbf{q}_{0}\right)+\phi_{t}\left(\mathbf{p}_{0}, \mathbf{q}_{0}\right)+E t\right)}\right|^{2}
\end{aligned}
$$

where $T$ is the total simulation time, $S_{t}\left(\boldsymbol{p}_{0}, \boldsymbol{q}_{0}\right)$ is the instantaneous action of the classically evolved trajectory $\left(\mathbf{p}_{t}, \mathbf{q}_{t}\right)$, and the phase-space integration is performed on the initial trajectory momenta $\boldsymbol{p}_{0}$ and positions $\boldsymbol{q}_{0}$. In eq $15,\left|\boldsymbol{p}_{t} \boldsymbol{q}_{t}\right\rangle$ are coherent states with the following expression in position representation $^{78}$

$$
\begin{aligned}
\left\langle\mathbf{x} \mid \mathbf{p}_{t} \mathbf{q}_{t}\right\rangle= & \left(\frac{\operatorname{det}(\gamma)}{\pi^{F}}\right)^{1 / 4} \exp \left[-\frac{1}{2}\left(\mathbf{x}-\mathbf{q}_{t}\right)^{\mathrm{T}} \gamma\left(\mathbf{x}-\mathbf{q}_{t}\right)\right. \\
& \left.+\mathrm{ip}_{t}^{\mathrm{T}}\left(\mathbf{x}-\mathbf{q}_{t}\right)\right]
\end{aligned}
$$

where $\gamma$ is an $N_{\text {vib }} \times N_{\text {vib }}$ diagonal matrix, whose elements are equal to the harmonic frequencies of the system. In eq 15 , $\phi_{t}\left(\boldsymbol{p}_{0}, \boldsymbol{q}_{0}\right)$ is the phase of the HHKK prefactor ${ }^{46,79}$

$$
\begin{aligned}
& \phi_{t}\left(\mathbf{p}_{0}, \mathbf{q}_{0}\right) \\
& \quad=\text { phase }\left[\sqrt{\frac{1}{2^{N_{v i b}}}\left|M_{\mathbf{q q}}(t)+\gamma^{-1} M_{\mathbf{p p}}(t) \gamma-\mathrm{i} M_{\mathbf{q p}}(t) \gamma+\mathrm{i} \gamma^{-1} M_{\mathbf{p q}}(t)\right|}\right]
\end{aligned}
$$

where $\boldsymbol{M}_{i j}$, with $i, j=\boldsymbol{p}, \boldsymbol{q}$, are the elements of the symplectic (monodromy or stability) $2 N_{\text {vib }} \times 2 N_{\text {vib }}$ matrix

$$
\mathbf{M}(t)=\left(\begin{array}{ll}
\mathbf{M}_{\mathbf{p p}}(t), & \mathbf{M}_{\mathbf{p q}}(t) \\
\mathbf{M}_{\mathbf{q p}}(t), & \mathbf{M}_{\mathbf{q q}}(t)
\end{array}\right)=\left(\begin{array}{ll}
\frac{\partial \mathbf{p}_{t}}{\partial \mathbf{p}_{0}}, & \frac{\partial \mathbf{p}_{t}}{\partial \mathbf{q}_{0}} \\
\frac{\partial \mathbf{q}_{t}}{\partial \mathbf{p}_{0}}, & \frac{\partial \mathbf{q}_{t}}{\partial \mathbf{q}_{0}}
\end{array}\right)
$$

Following Hamilton's equations, the time-evolution of $\mathbf{M}(t)$ is

$$
\frac{\mathrm{d}}{\mathrm{d} t} \mathbf{M}(t)=\mathbf{A} \cdot \mathbf{M}(t)
$$

where

$$
\mathbf{A}=\left(\begin{array}{ll}
0 & -\mathbf{H}(t) \\
1 / \mathbf{m} & 0
\end{array}\right)
$$

and where $\mathbf{H}(t)$ is the Hessian matrix at time $t$. Thus, it is necessary for an accurate Hessian approximation to have an accurate $\mathbf{M}(t)$ matrix and an accurate vibrational power spectrum. We monitored the accuracy of $\mathbf{M}(t)$ by exploiting its symplectic property and checking the deviation of its determinant (or better, the determinant of the positive-definite matrix $\left.\mathbf{M}^{\mathrm{T}}(t) \mathbf{M}(t)\right)$ from unity.

To beat the curse of dimensionality, we reduced the phase space integration of eq 15 to a few, or just one, trajectory simulations, where each trajectory starts from the global minimum and with an energy equal to the harmonic vibrational energy level that one is looking for. ${ }^{80}$ This method is called multiple coherent SCIVR (MC SCIVR), ${ }^{81,82}$ and it exploits the fact that during the simulation, the delocalization of the coherent states will account for anharmonicity and reproduce the anharmonic vibrational peak position. ${ }^{14,15,83-95}$ The method also allows to identify each mode contribution by selecting a suitable combination of coherent states obtained by changing the sign of the momentum part of the coherent state. ${ }^{96}$ This method has been recently further improved by introducing the DC SCIVR method. The DC basic idea is to calculate the full-dimensional spectrum as the composition of subdimensional ones using eq 15 but with reduced dimensionality phase space quantities. In eq 15 , the potential, which is a part of the action, is the only quantity that cannot be exactly projected in a reduced dimensionality space. For this reason, we have introduced the following approximation for the partial $M$-dimensional spectrum

$$
\begin{aligned}
\tilde{S}_{t}\left(\tilde{\mathbf{p}}_{0}, \tilde{\mathbf{q}}_{0}\right)= & \int_{0}^{t} \mathrm{~d} t^{\prime}\left[\frac{1}{2} \tilde{\mathbf{p}}_{t^{\prime}}^{\mathrm{T}} \tilde{\mathbf{p}}_{t^{\prime}}-\left(V\left(\tilde{\mathbf{q}}_{t^{\prime}}, \mathbf{q}_{t^{\prime}}{ }^{\left(N_{\mathrm{vib}}-M\right)}\right)\right.\right. \\
& \left.\left.-V\left(\tilde{\mathbf{q}}_{\mathrm{eq}}, \mathbf{q}_{t^{\prime}}{ }^{\left(N_{\mathrm{vib}}-M\right)}\right)\right)\right]
\end{aligned}
$$

where the tilde $\sim$ indicated a $M$-dimension quantity, $\mathrm{V}\left(\tilde{\mathbf{q}}_{t^{\prime}}, \mathbf{q}_{t^{\prime}}{ }^{\left(N_{v i b}-M\right)}\right)$ is the full dimensional potential, and $\mathrm{V}$ $\left(\tilde{\mathbf{q}}_{\text {eq }}, \mathbf{q}_{t^{\prime}}{ }^{\left(N_{\text {vib }}-M\right)}\right)$ is the one obtained by fixing at equilibrium the coordinates in the $M$-dimensional subspace. This approach has been successfully applied to several high-dimensional complex systems, including fluxional ones, like small water clusters ${ }^{97}$ and the protonated water dimer. ${ }^{98}$ When combined with MC SCIVR by projecting few full-dimensional classical trajectories into sub-dimensional phase space components, we obtain the MC-DC SCIVR, which can deal with very high-dimensional systems. Notable applications of MC-DC SCIVR include dipeptide derivatives, ${ }^{99}$ nucleobases ${ }^{100}$ and nucleosides, ${ }^{101}$ water clusters up to $\left(\mathrm{H}_{2} \mathrm{O}\right)_{23},{ }^{102,103}$ and molecules adsorbed on titania surfaces. ${ }^{104}$ In the DC SCIVR method, one needs to properly partition the full-dimensional vibrational space. One can reach this goal by coarse-graining the time-averaged Hessian matrix or by splitting the Jacobian (monodromy) matrix $\mathbf{M}(t)$ in square blocks, such that the determinant of each block is as close as possible to 1 , in partial satisfaction of Liouville's theorem. In either case, the result is a block diagonalized matrix, where each block represents a vibrational subspace. If one chooses to use the Jacobian approach, the probability graph-evolutionary algorithm (PG-EA) that we recently reported is the way to go. ${ }^{105}$ The PG-EA algorithm uses a cluster graph representation of the molecule's normal modes, where connected modes are within the same subspace. Such a representation is particularly advantageous because it is invariant with respect to the permutation of modes and subspaces.

\section{RESULTS}

In this section, we present simulations of growing complexity, starting from the small molecular systems $\mathrm{H}_{2} \mathrm{O}, \mathrm{HCOH}$, and $\mathrm{CH}_{4}$, going to the smallest prototype of peptide bond [trans $\mathrm{N}$ methylacetamide (NMA)], up to a small synthetic peptide ( $N$ acetyl-L-phenylalaninyl-L-methionine amide), which is composed of 46 atoms and 132 vibrational degrees of freedom. All simulations consist of a single 3000 time-step constant energy $(N V E)$ classical trajectory with a 10 a.u. constant time-step. The initial conditions are chosen according to the MC-DC SCIVR recipe described above. The classical equations of motion are integrated using a four-order symplectic integrator. ${ }^{106} \mathrm{We}$ employed precomputed $\mathrm{PES}$ for $\mathrm{H}_{2} \mathrm{O}$, ${ }^{107}$ $\mathrm{HCOH},{ }^{108} \mathrm{CH}_{4},{ }^{109}$ and NMA, ${ }^{110}$ while the $\mathrm{N}$-acetyl-Lphenylalaninyl-L-methionine amide (Ac-Phe-Met- $\mathrm{NH}_{2}$ ) molecule was simulated on-the-fly by direct $a b$ initio $\mathrm{MD}$ at the level of DFT-B3LYP-D/6-31G* theory. The PES derivatives are 
Table 1. Accuracy and Computational Time for Different Hessian Approximation Methods ${ }^{a}$

\begin{tabular}{|c|c|c|c|c|c|c|c|}
\hline molecule & \#Hessians & method & $10^{2} \sigma_{\mathrm{Hess}}$ & relative $\sigma_{\mathrm{Hess}}{ }^{b}$ & method cpu-time & Hessians cpu-time & total cpu-time \\
\hline \multirow[t]{4}{*}{$\mathrm{H}_{2} \mathrm{O}$} & 150 & NGas & 0.539 & 1.00 & 19.314 & 0.197 & 19.51 \\
\hline & 150 & $\mathrm{DBH}(\rho=2.59)$ & 0.728 & 1.35 & 0.355 & & 0.55 \\
\hline & 150 & Bofill & 2.336 & 4.33 & 0.148 & & 0.346 \\
\hline & 3000 & all Hessians & 0.000 & NA & 0.000 & 3.947 & 3.95 \\
\hline \multirow[t]{4}{*}{$\mathrm{HCOH}$} & 150 & NGas & 0.612 & 1.00 & 19.395 & 0.356 & 19.75 \\
\hline & 150 & $\mathrm{DBH}(\rho=8.22)$ & 0.824 & 1.34 & 0.329 & & 0.69 \\
\hline & 150 & Bofill & 1.570 & 2.56 & 0.160 & & 0.52 \\
\hline & 3000 & all Hessians & 0.000 & NA & 0.000 & 7.115 & 7.12 \\
\hline \multirow[t]{4}{*}{$\mathrm{CH}_{4}$} & 150 & NGas & 0.732 & 1.00 & 18.923 & 0.492 & 19.42 \\
\hline & 150 & $\mathrm{DBH}(\rho=7.95)$ & 1.000 & 1.37 & 0.345 & & 0.84 \\
\hline & 150 & Bofill & 2.231 & 3.05 & 0.192 & & 0.68 \\
\hline & 3000 & all Hessians & 0.000 & NA & 0.000 & 9.835 & 9.84 \\
\hline \multirow[t]{4}{*}{ NMA } & 150 & NGas & 0.447 & 1.00 & 22.582 & 12.842 & 35.42 \\
\hline & 150 & $\mathrm{DBH}(\rho=21.15)$ & 0.490 & 1.09 & 0.499 & & 13.34 \\
\hline & 150 & Bofill & 0.935 & 2.09 & 0.262 & & 13.10 \\
\hline & 3000 & all Hessians & 0.000 & NA & 0.000 & 256.834 & 256.83 \\
\hline \multirow[t]{4}{*}{ Ac-Phe-Met- $\mathrm{NH}_{2}$} & 298 & NGas & 0.059 & 1.00 & 27.667 & $7620.819^{c}$ & $7621.28^{c}$ \\
\hline & 298 & $\mathrm{DBH}(\rho=11.9)$ & 0.059 & 1.00 & 2.697 & & $7620.86^{c}$ \\
\hline & 312 & Bofill & 0.153 & 2.58 & 2.030 & $7978.845^{c}$ & $7978.88^{c}$ \\
\hline & 2500 & all Hessians & 0.000 & NA & 0.000 & $63,933.049^{c}$ & $63,933.05^{c}$ \\
\hline
\end{tabular}

${ }^{a}$ First column is the molecule, the second column is the number of exact Hessian calculations, the third column is the Hessian approximation method, the fourth column is the error according to eq 13, the fifth column is the relative error respect to the NGas method, the sixth column is the cpu-time for each method, the seventh column is the cpu-time for the exact Hessian evaluation, and the last column is the total computational time. All times are in seconds, except explicitly indicated. For each molecule, the NGas, the DBH at threshold $\rho$, and the CFD (Bofill) methods are tested. The "all Hessians" label is for Hessian calculations at each time-step, that is, without any approximation. ${ }^{b}$ Defined as the error of the method divided by the error of the NGas method. ${ }^{c}$ Core hours (average of core hours necessary for the computation).

computed by finite differences with a fixed displacement of $10^{-3}$ a.u. In the case of the trans NMA calculation, we employ an analytical gradient PES, ${ }^{110,111}$ and the Hessian matrix is computed by the finite difference of the gradient. The NGas method has been optimized using 150 neurons for the simulation of $\mathrm{H}_{2} \mathrm{O}, \mathrm{HCOH}, \mathrm{CH}_{4}$, and $\mathrm{NMA}$, and 300 neurons are employed in the case of Ac-Phe-Met- $\mathrm{NH}_{2}$. The number of learning epochs and the $\alpha_{\text {init }} \alpha_{\text {final }}, \lambda_{\text {init }}, \lambda_{\text {final }}$ parameters are kept fixed, as described in the Methods section. We performed all computations reported here on a computer laptop using a single core [Intel(R) Core(TM) i7-4510U CPU@2.00GHz, with less than $16 \mathrm{~GB}$ of available memory] with the exception of Ac-Phe-Met- $\mathrm{NH}_{2}$, whose Hessians have been computed on the group computer cluster using 10 cores [Intel(R) Xeon(R) CPU E5-2660 v3@2.60GHz 125Gb] per Hessian matrix.

Hessian Approximation Accuracy. To test the accuracy of each method, we performed calculations where 150 Hessians (300 in the case of Ac-Phe-Met- $\mathrm{NH}_{2}$ ) out of 3000 time-steps (2500 in the case of Ac-Phe-Met- $\mathrm{NH}_{2}$ ) are calculated explicitly, that is, from the PES or the electronic structure, and the remaining ones are approximated. In others words, exact Hessians are estimated every 20 (about 8 in the case of Ac-Phe-Met- $\mathrm{NH}_{2}$ ) MD time-steps, and all others are approximated. The deviations of the approximated Hessians from the exact ones are estimated using eq 13. Notice that, although the Hessians in eq 13 are in Cartesian coordinates, we employ normal mode coordinates in the DBH and NGas methods to locate the optimal configurations.

Table 1 reports the results of this test for each molecule, and it shows that the computational time of the Hessian matrix calculation is the simulation bottleneck when evaluated by $a b$ initio methods. Actually, when using a precomputed PES, the time required for the NGas algorithm iterations is roughly of the same order of magnitude of evaluating the Hessian for each trajectory configuration. To appreciate the advantage of the approximation schemes in terms of cpu-time, one has to reach the 30 degrees of freedom of the NMA molecule. However, even in this case, the use of analytical gradients provided by the precomputed $\mathrm{PES}^{110}$ accelerates the Hessian matrix estimation and keeps the option to evaluate all Hessians along the trajectory viable. We can see a clear advantage of the approximation methods only when dealing with Ac-Phe-Met$\mathrm{NH}_{2}$, which we simulated on-the-fly. In this case, the evaluation of a single Hessian ( $\mathrm{SH}$ ) matrix takes about $3 \mathrm{~h}$ with NWChem package ${ }^{112}$ on a 10 core $[\operatorname{Intel}(\mathrm{R}) \operatorname{Xeon}(\mathrm{R})$ CPU E5-2660 v3 @ 2.60GHz 125Gb] node, and the NGas and $\mathrm{DBH}$ methods become, in this case, the only viable option. Table 1 reports also in the fourth column the error $\sigma_{\text {Hess }}$ for each method with respect to the all-Hessian evaluation. We notice that the NGas method is as accurate as the $\mathrm{DBH}$ one in the case of Ac-Phe-Met- $\mathrm{NH}_{2}$. In the fifth column of Table 1, the relative $\sigma_{\text {Hess }}$ shows how each method compares with the NGas in terms of accuracy. We see that by using the NGas method, we can decrease the error by about $26 \%$ for small molecular systems, while the NGas error is comparable with the DBH method in the cases of the NMA and Ac-Phe-Met$\mathrm{NH}_{2}$ molecules. We can understand this trend, considering that the higher the number of degrees of freedom, the less often the trajectory visits the same phase space region. In these cases, the NGas method provides a solution that is very similar to the $\mathrm{DBH}$ one since neurons are distributed along the trajectory and basically coincide with the molecular geometries at which Hessian matrices are calculated according to the $\mathrm{DBH}$ approach.

Overall, we can observe that the ratio of computational time versus the number of degrees of freedom is almost constant for 
all methods, and it increases moderately only in the case of the Ac-Phe-Met- $\mathrm{NH}_{2}$ system. This is mainly due to the time required to store and copy the trajectory. At this stage, we cannot assert what will happen for even higher-dimensional systems. However, we still can test the robustness and stability of each method by decreasing the number of PES or $a b$ initio Hessian entries. In this way, we can also better understand which are the minimum number of Hessian evaluations necessary for obtaining an accurate estimate. We focus on the Ac-Phe-Met- $\mathrm{NH}_{2}$ system and on the NGas and DBH approximations. Table 2 reports the values of $\sigma_{\text {Hess }}$ of eq 13 for

Table 2. Hessian Element MAE from Eq $13\left(\sigma_{\text {Hess }}\right)$ for the NGas and DBH Methods by Varying the Number of Exact Hessian Evaluations Indicated in the Second Column in the Case of the Ac-Phe-Met- $\mathrm{NH}_{2}$ Molecule

\begin{tabular}{|c|c|c|c|c|}
\hline \multirow[b]{2}{*}{ method } & \multirow[b]{2}{*}{ \#Hessians } & \multicolumn{2}{|c|}{$10^{2} \sigma_{\mathrm{Hess}}$} & \multirow{2}{*}{$\frac{\text { relative } \sigma_{\text {Hess }}{ }^{b}}{\tilde{\mathrm{q}} \cup \nabla \tilde{\mathrm{q}}^{a}}$} \\
\hline & & $\tilde{q}^{a}$ & $\tilde{\mathrm{q}} \cup \nabla \tilde{\mathrm{q}}^{a}$ & \\
\hline NGas & 25 & 0.372 & 0.260 & 1.00 \\
\hline $\mathrm{DBH}(\rho=55.0)$ & 25 & 0.319 & & 1.23 \\
\hline NGas & 50 & 0.357 & 0.219 & 1.00 \\
\hline $\mathrm{DBH}(\rho=40.0)$ & 50 & 0.267 & & 1.21 \\
\hline NGas & 100 & 0.157 & 0.153 & 1.00 \\
\hline $\mathrm{DBH}(\rho=27.3)$ & 100 & 0.167 & & 1.09 \\
\hline NGas & 200 & 0.090 & 0.089 & 1.00 \\
\hline $\mathrm{DBH}(\rho=17.3)$ & 200 & 0.090 & & 1.01 \\
\hline
\end{tabular}

${ }^{a}$ The columns " $\tilde{q}$ " and “ $\tilde{q} \cup \nabla \tilde{q}$ " refer to different NGas training spaces, as described in the text, while the last column reports the relative error with respect to the best NGas estimate. ${ }^{b}$ Defined as the error of the method divided by the error of the NGas method.

the two methods for the different exact Hessian evaluation times reported in the second column. Clearly, the more the $a b$ initio Hessians are computed, the smaller the approximation error is, as reported in the third column. If the NGas and DBH errors are compared for about 200 exact Hessian evaluations, $\mathrm{DBH}$ is more and more accurate as the number is significantly reduced down to 25 . We think that this poor performance of the NGas method is due to the fact that, given the extremely low numbers of Hessians provided, the neuron locations are not representatives of their trajectory neighborhood. In other words, when the system conformation is averaged over many ones, the result may be very different from the actual conformations visited along the classical trajectory. To improve and going beyond this limitation, we use an extended set of variables for the neurons' space, which includes also the potential gradients in the NGas training process. While the original neurons are identified by a set of normal mode molecular coordinates of the type $\tilde{\mathbf{q}}=\left(\tilde{q}_{1}, \ldots, \tilde{q}_{N_{\text {vib }}}\right)$ in the improved version, the vector which identifies the neuron includes the energy gradient coordinates as well, $\tilde{\mathbf{q}} \cup \nabla \tilde{\mathbf{q}}=\left(\tilde{q}_{1}, \ldots, \tilde{q}_{N_{\text {vib }}}, \frac{\partial V\left(\tilde{q}_{1}\right)}{\partial \tilde{q}_{1}}, \ldots, \frac{\partial V\left(\tilde{q}_{N_{\text {vib }}}\right)}{\partial \tilde{q}_{N_{\text {vib }}}}\right)$. This extended neuron set of variables accounts for the PES slope in addition to the molecular positions. In this way, unrealistic conformations with huge internuclear forces (and consequently large Hessian elements) are excluded in favor of more realistic conformations. The improved results are reported in the last column of Table 2. The extended NGas is always more accurate not only with respect to the original NGas method but also to the $\mathrm{DBH}$ method, in particular, for the cases when there are few exact Hessian estimates. We observe again that when the number of neurons is increased, these are allowed to have a neighboring trajectory segment that is a straight line, and thus the NGas and DBH methods become alike.

Although the NGas method seems to provide a small improvement with respect to the $\mathrm{DBH}$ one for the larger systems, that is, NMA and Ac-Phe-Met- $\mathrm{NH}_{2}$, we can prove that it can reach an accuracy comparable to that one observed for the smaller systems. In fact, both NGas and DBH methods approximate only the regions of configurational space that are close to the trajectory since they are based on the distance from neighboring geometries. Hence, if we employ 150 neurons to approximate a 3000 step trajectory, each Voronoi cell contains on average 20 geometries and the related Hessians. When the system becomes larger, we expect these geometries to be visited within the same portion of the trajectory. This is the reason why DBH and NGas methods provide more and more similar results as the system size grows. However, things are different if we use an ensemble of $\mathrm{MD}$ trajectories because in this case, it is very likely that trajectories cross and overlap significantly, as in a tangle of strings. Table 3 reports the numerical results of two ensembles of trajectories.

Table 3. Hessian Element MAE of Eq $13\left(\sigma_{\text {Hess }}\right)$ for the NMA Molecule Using the NGas and DBH Methods Obtained by Varying Either the Total Number of configurations (second Column) or the Number of $a b$ initio Hessians (Third Column)

$\begin{array}{lcccc}\text { method } & \begin{array}{c}\text { configurations } \\ (\# \text { trajectories } \times \text { \#steps })\end{array} & \text { \#Hessians } & 10^{2} \sigma_{\text {Hess }} & \begin{array}{c}\text { relative } \\ \sigma_{\text {Hess }}\end{array} \\ \text { NGas } & 100 \times 1000 & 999 & 1.33 & 1.00 \\ \text { DBH } & 100 \times 1000 & 1008 & 1.64 & 1.23 \\ (\rho=54.7) & 500 \times 1000 & 1000 & 1.52 & 1.00 \\ \text { NGas } & 500 \times 1000 & 999 & 1.94 & 1.28 \\ \text { DBH } & & & & \\ (\rho=67.6) & 100 \times 1000 & 999 & 1.33 & 1.00 \\ \text { NGas } & 100 \times 1000 & 2049 & 1.34 & 1.01 \\ \text { DBH } & & & & \\ (\rho=45.6) & 500 \times 1000 & 1000 & 1.52 & 1.00 \\ \text { NGas } & 500 \times 1000 & 6082 & 1.56 & 1.03 \\ \text { DBH } & & & & \end{array}$

${ }^{a}$ Defined as the error of the method divided by the error of the NGas method

The trajectory initial conditions were sampled from the Husimi distribution in phase space centered at the equilibrium values. We notice that the trajectories originated by this distribution are spread in energy values, in contrast to previous simulations, and the errors in the Hessian matrix are inevitably higher. In the upper part of Table 1, the NGas method provides a smaller value of $\sigma_{\text {Hess }}$ for the same number of $a b$ initio Hessians employed in the DBH simulation. In the lower part of the table, the same average error in the Hessian matrix is reached only when $\mathrm{DBH}$ employs more than 6 times $a b$ initio Hessians than the NGas method. With about 500 thousand geometries and one thousand neurons, our implementation of the NGas method takes its toll, and the training of the NGas takes about $7 \mathrm{~h}$ to be optimized compared to the $50 \mathrm{~min}$ required by $\mathrm{DBH}$. However, if one takes into account the $\sim 10$ min per core [Intel(R) Xeon(R) CPU E5-2660 v3@2.60GHz $125 \mathrm{~Gb}]$ that it takes to compute the $a b$ initio Hessian matrix of NMA at DFT-B3LYP/6-31G* level of theory, it is still 


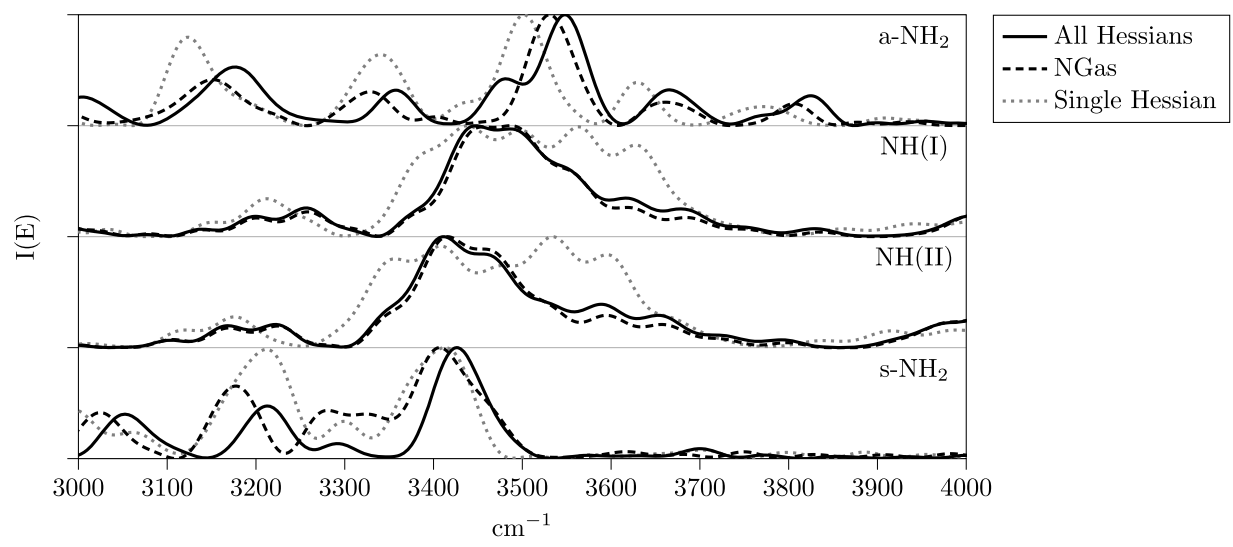

Figure 3. Spectroscopic Hessian accuracy test for the $\mathrm{NH}_{2}$ stretches in the amide group of the Ac-Phe-Met- $\mathrm{NH}_{2}$ peptide. The dotted line is for the $\mathrm{SH}$ approximation; ${ }^{115}$ the dashed line is for the NGas approximation including the gradient information with 200 neurons and $200 a b$ initio Hessian calculations. The continuous line, which is labeled as "all Hessians," reports the simulation where all Hessians are obtained from ab initio calculations.

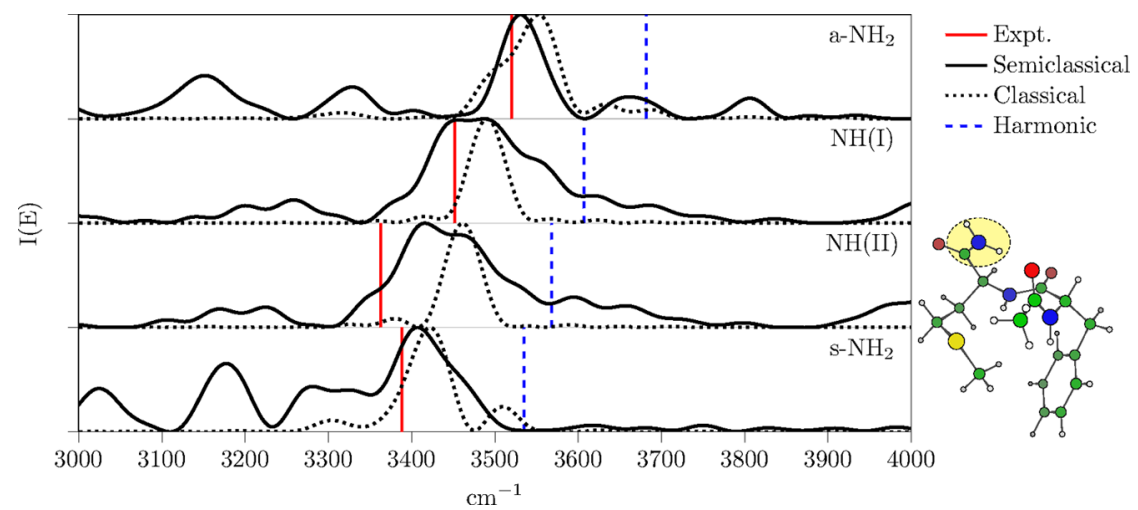

Figure 4. Ac-Phe-Met- $\mathrm{NH}_{2}$ amide group related vibrational stretching power spectra. Vertical continuous sticks are the experimental values, ${ }^{114}$ while vertical dashed sticks are the harmonic approximation frequencies. Continuous lines are for MC-DC SCIVR simulation using the Hessian NGas approximation and dotted lines are for the quasi-classical simulation on the same $a b$ initio potential (see main text).

convenient to use the NGas method. Finally, the $\sigma_{\text {Hess }}$ values reported in Table 3 are rather large as we enforced less than one $a b$ initio Hessian matrix every 100 Hessians, which is quite a drastic setup.

Spectroscopic Simulations. One may wonder which level of Hessian approximation accuracy is requested in MD applications and how important the choice of the approximation method is. To reply to this question, we decided to employ our approximate Hessians for the integration of eq 19 and the calculation of the power spectrum using eq 15 . Specifically, we simulated the full-dimensional vibrational power spectrum of the small Ac-Phe-Met- $\mathrm{NH}_{2}$ peptide using a single on-the-fly $a b$ initio trajectory with our MC-DC SCIVR method, described in the Semiclassical Initial Value Representation Vibrational Spectroscopy section. In the DC strategy, we need to find a vibrational space subdivision, which is the result of a trade-off between spectroscopic accuracy and feasibility. Too high-dimensional vibrational subspaces are not practical, but too low-dimensional ones may turn out to be a drastic approximation. For these reasons, we performed a preliminary coarse-graining of the time-averaged Hessian matrix by fixing to zero all elements smaller than $8.0 \times 10^{-6}$ a.u. ${ }^{113}$ In this way, after conveniently permuting rows and columns, we obtained a block diagonal matrix whose 23dimensional subspace contains all stretching modes of the amine group we are interested in. These are denominated as
$\mathrm{sNH}_{2}$ (mode number 129), $\mathrm{NH}(\mathrm{II})$ (130), $\mathrm{NH}(\mathrm{I})$ (131), and $\mathrm{aNH}_{2}$ (132). We focus on these fundamentals because their experimental values are available for comparison. ${ }^{114}$ This subspace is further decomposed into smaller subspaces using our PG-EA algorithm. ${ }^{105}$ The stretches we are interested in are highlighted in bold in the normal mode subspaces \{ $\begin{array}{ll}10 & 30\end{array}$

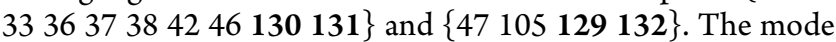
numbers are assigned according to the harmonic frequency values, where smaller numbers mean lower harmonic frequency values. Both subspaces contain floppy modes. In particular, the first subspace contains several floppy modes, and we expect that the partial spectra of the $\mathrm{NH}(\mathrm{II})$ (130) and $\mathrm{NH}(\mathrm{I})$ (131) modes will embody several combination features of these stretches with floppy modes.

Figure 3 shows the power spectra of the selected amide group stretching modes using different Hessian approximations. On each panel is reported the signal of each mode after a suitable combination of coherent states. ${ }^{96}$ Continuous lines are for MC-DC SCIVR simulations where Hessians have been calculated at each time-step and are labeled as "all Hessians." Dashed lines are for our NGas approximation presented above, that is, with the inclusion of the gradients in the set of neuron variables. The dotted line is the so-called "SH" approximation, ${ }^{115}$ where the Hessian is constant and it is equal to the equilibrium geometry one. The NGas simulation is very similar to the exact, especially for the higher-dimensional subspace. 
However, the SH approximation is quite good if one takes into account how drastic the approximation is. Nevertheless, the main problem of the $\mathrm{SH}$ approximation is that for the higherdimensional subspace containing the $\mathrm{NH}(\mathrm{I})$ and $\mathrm{NH}(\mathrm{II})$ stretches, it does not allow for a definitive assignment, while in the case of the NGas spectra, a main peak is present, despite the numerous overtone side peaks. We can confirm that these side peaks of smaller intensities are of the type of overtones or combination bands by comparing the NGas spectra with the classical ones in Figure 4.

Quasi-classical spectra are obtained by Fourier transforming the velocity-velocity correlation function of a constant energy trajectory (NVE), which is the same one employed for the MC-DC SCIVR calculations, that is, trajectories starting from the equilibrium geometry and with kinetic energy equals to the harmonic zero-point energy (ZPE). While these types of classical simulations provide frequency values with anharmonic corrections because the $a b$ initio trajectory accounts for the shape of the PES, these values are restricted only to the fundamental transition frequencies and higher harmonics. Instead, semiclassical simulations, such as MC-DC SCIVR, provide the full collection of eigenvalues as in eq 14, and all type of transition frequencies can be obtained by the difference between the eigenvalues. Thus, the semiclassical power spectrum includes not only the fundamental frequencies but also anharmonic overtones, combination bands, and the ZPE value on an absolute scale. For these reasons, the MC-DC SCIVR spectra of Figure 4 (continuous lines) present several more spectroscopic features than the classical ones (dashed lines). However, it is still possible to compare the two of them with the experiments on the fundamental frequency values. The comparison is reported in Table 4 and Figure 4, where the

Table 4. Selected Amide Group Vibrational Stretching Fundamentals of the Ac-Phe-Met- $\mathrm{NH}_{2}$ Peptide at Different Levels of Approximation ${ }^{a}$

$\begin{array}{lcrrrrr}\text { modes } & \text { all Hessians } & \text { NGas } & \text { DBH }^{16} & \text { classical } & \text { harmonic } & \exp ^{114} \\ \mathrm{aNH}_{2} & 3548 & 3530 & 3490 & 3552 & 3682 & 3520 \\ \mathrm{NH}(\mathrm{I}) & 3448 & 3456 & 3480 & 3490 & 3607 & 3452 \\ \mathrm{NH}(\mathrm{II}) & 3412 & 3416 & 3300 & 3461 & 3568 & 3363 \\ \mathrm{sNH}_{2} & 3426 & 3406 & 3360 & 3422 & 3535 & 3388 \\ \mathrm{MAE} & 29 & 21 & 37 & 51 & 167 & 0.0\end{array}$

${ }^{a}$ The first column reports the type of stretch, the second reports the MC-DC SCIVR frequencies without any Hessian approximation, the third and the fourth columns, respectively, report the NGas and the DBH approximated Hessians semiclassical frequency values, the fifth column is the quasi-classical frequencies of vibration, the sixth column is the harmonic results, and the last column shows the experimental values. ${ }^{114}$ In the last row, the MAE with respect to the experimental values is reported for each method.

experimental values ${ }^{114}$ are reported as a red continuous stick spectrum, while the harmonic estimates are the dashed blue sticks. Overall, we can observe in Figure 4 that the semiclassical simulations present broader peaks than the classical ones. The classical peak width is what is expected from the Fourier transform of a $\sim 0.73$ ps simulation. We do not pursue longer trajectories because the quantum accuracy of the semiclassical approximation would deteriorate for longer simulations. We also decided to not apply any artificial exponential constant decay (Gaussian filter) to avoid any sort of biasing. In Figure 4, the semiclassical signals are broader in the case of the $\mathrm{NH}(\mathrm{I})$ and $\mathrm{NH}(\mathrm{II})$ stretches, as expected, because of the numerous strongly coupled floppy modes. Specifically, the more intense peaks in the $\mathrm{NH}(\mathrm{I})$ and $\mathrm{NH}(\mathrm{II})$ panels represent the convolution of a series of overtones coupled to the numerous floppy modes, while the other side peaks, which are absent in the classical spectrum, are a combination or overtone bands of other modes. In fact, both the subspace subdivision and the filtering process provided by the combination of coherent states $^{98}$ can only partially filter the numerous eigenvalues which are present in a given energy window of a 132dimensional power spectrum. Clearly, in Figure 4, these side peaks are less intense at higher frequencies because the trajectory energy shell is at the level of the harmonic ZPE value, where the Fourier transformed coherent state is centered.

Table 4 summarizes the results in Figure 4 with the additional results of the semiclassical MC-DC SCIVR simulation obtained using the Hessian database approximation. ${ }^{16}$

The comparison between different levels of calculation shows that classical and semiclassical results are systematically more accurate than the harmonic ones, while the semiclassical ones are further more accurate with respect to the classical ones. The semiclassical reference is reported in the second column of Table 4, where the calculations have been performed without any Hessian approximation but using directly the $a b$ initio values. The third and fourth columns report, respectively, the NGas and the DBH approximated Hessian semiclassical values. For the NGas simulation, 200 neurons and $200 a b$ initio Hessians have been employed, while the DBH results are obtained with $300 a b$ initio Hessians. ${ }^{16}$ At this level of comparison, we think it is not possible to assert which of the Hessian approximations, either the NGas or the $\mathrm{DBH}$ one, is more appropriate for spectroscopic analyses with the MC-DC SCIVR method. Actually, the NGas MAE with respect to the experimental values in Table 4 is slightly smaller than calculating all $a b$ initio Hessians. This is clearly due to a compensation of effects, which include the level of $a b$ initio theory. Eventually, given the NGas and DBH MAE of Table 4, both of them are accurate enough for semiclassical calculations, considering that any semiclassical simulation strongly depends on the level of $a b$ initio theory and that the Fourier transform broadening is about $\sim 20 \mathrm{~cm}^{-1}$ for a typical semiclassical trajectory simulation, where the total time is on the order of picoseconds.

\section{CONCLUSIONS}

Given the importance of an accurate method for approximating instead of calculating the Hessian matrix during MD simulations, we have investigated the possibility to employ a slightly customized NGas algorithm that allows us to compute the Hessian matrix of the potential energy along a MD simulation. After presenting the method, we have tested its accuracy compared to other algorithms already present in the literature. ${ }^{14-16}$ Then, we applied it to speeding up the calculation of semiclassical spectra, where the Hessian calculation is mandatory at each MD time-step. We find that the NGas algorithm can be $\sim 20 \%$ more accurate than other methods for simulations of molecular systems whose trajectories overlap and cross significantly. Furthermore, it appears that the NGas method may require far fewer $a b$ initio Hessian calculations to provide the same accuracy as competitive methods. However, some caveats must be taken 
into account. First of all, if one aims to study a single short trajectory of a large molecular system (such as Ac-Phe-Met$\mathrm{NH}_{2}$ ), it appears that the NGas method is just as accurate as the $\mathrm{DBH}$ approach that our group presented recently. ${ }^{16}$ As a matter of fact, in such cases, the NGas method provides a solution that is similar to that proposed by $\mathrm{DBH}$. Furthermore, if the user can afford to compute only very few Hessian calculations along a nonoverlapping trajectory, it is recommended to add gradients of the potential to the NGas training set. This set up is slightly more robust than the DBH method with respect to the number of $a b$ initio Hessians. Second, while at high dimensions, all methods scale favorably, the NGas method would suffer from longer simulations and higher number of neurons. However, we expect that this feature should still compensate for the time spent for the $a b$ initio calculation of all Hessians. We did not pursue the simulations of molecular systems significantly larger than Ac-Phe-Met- $\mathrm{NH}_{2}$ because the Hessian calculations at each time-step would be out of reach for standard computational power. The third caveat is that the DBH method can also be performed on-thefly, while the NGas one is necessarily a postprocessing method. This means that in the $\mathrm{DBH}$ method, the number of $a b$ initio Hessian calculations can be automatically determined during the dynamics if one applies the method to the available database at each time-step and increment the database during the dynamics, while in the case of the NGas method, it has to be fixed a priori. The last caveat is that the $\mathrm{DBH}$ parameter $\rho$ is system-dependent. Also, $\rho$ ensures that the approximated Hessians are close enough to the trajectory, but it does not allow to control the number of Hessians to compute. On the other hand, the NGas method requires as input the number of Hessians one is willing to compute, but it does not assure that the neuron locations would be close enough to the trajectory. Nevertheless, both these shortcomings can be mended by a preliminary trial and error calculation. Eventually, for semiclassical spectroscopic calculations, we conclude that both methods are accurate. We also tested the $\mathrm{SH}$ approximation and confirm that this choice should be avoided or employed as a preliminary calculation together with a classical power spectrum calculation. Finally, in this work, we have also shown that our DC SCIVR technique implemented by reasonable approximations can allow for power spectrum calculations with the inclusion of quantum nuclear features of systems as large as small peptides. As a future perspective, our NGas method could be interfaced with methods that generate a local fit of the potential, such as the GPR-PES method. ${ }^{22}$ In fact, the trajectory geometries within a Voronoi cell can be used to train a GPR model and better approximate the Hessian matrix within the same cell. This approach would allow for more reliable Hessian estimates within the current limitations of applications of the GPR-PES methodology.

\section{CODE AVAILABILITY}

The codes developed for this work are freely available on github at: https://github.com/ganmichele/hessapprox.

\section{ASSOCIATED CONTENT}

\section{SI Supporting Information}

The Supporting Information is available free of charge at https://pubs.acs.org/doi/10.1021/acs.jctc.1c00707.

Pseudocodes for NGas, DBH, and CFD-Bofill methods, scatter plots of neurons and trajectory geometries for
$\mathrm{H}_{2} \mathrm{O}$ and $\mathrm{HCOH}$ molecules, and a plot of the Hessian matrix element absolute error versus the trajectory step(PDF)

NGas intermediate steps on a 2D plane (MP4)

\section{AUTHOR INFORMATION}

\section{Corresponding Author}

Michele Ceotto - Dipartimento di Chimica, Università degli Studi di Milano, 20133 Milano, Italy; @orcid.org/0000-

0002-8270-3409; Email: michele.ceotto@unimi.it

\section{Author}

Michele Gandolfi - Dipartimento di Chimica, Università degli Studi di Milano, 20133 Milano, Italy; 이이.org/00000001-8319-3773

Complete contact information is available at:

https://pubs.acs.org/10.1021/acs.jctc.1c00707

\section{Notes}

The authors declare no competing financial interest.

\section{ACKNOWLEDGMENTS}

The authors thank Dr. Riccardo Conte for useful discussions. The authors acknowledge financial support from the European Research Council (grant agreement no. (647107)-SEMICOMPLEX-ERC-2014-CoG) under the European Union's Horizon 2020 research and innovation programme and from the Italian Ministery of Education, University, and Research (MIUR) (FARE programme R16KN7XBRB- project QURE).

\section{REFERENCES}

(1) Lourderaj, U.; Song, K.; Windus, T. L.; Zhuang, Y.; Hase, W. L. Direct dynamics simulations using Hessian-based predictor-corrector integration algorithms. J. Chem. Phys. 2007, 126, 044105.

(2) Bakken, V.; Millam, J. M.; Bernhard Schlegel, H. Ab initio classical trajectories on the Born-Oppenheimer surface: Updating methods for Hessian-based integrators. J. Chem. Phys. 1999, 111, 8773-8777.

(3) Hratchian, H. P.; Schlegel, H. B. Using Hessian updating to increase the efficiency of a Hessian based predictor-corrector reaction path following method. J. Chem. Theory Comput. 2005, 1, 61-69.

(4) Schlegel, H. B. Estimating the Hessian for gradient-type geometry optimizations. Theor. Chim. Acta 1984, 66, 333-340.

(5) Schlegel, H. B. Modern Electronic Structure Theory: Part I; World Scientific, 1995; pp 459-500.

(6) Kindt, J. T.; Schmuttenmaer, C. A. Far-infrared absorption spectra of water, ammonia, and chloroform calculated from instantaneous normal mode theory. J. Chem. Phys. 1997, 106, $4389-4400$.

(7) Imoto, S.; Marx, D. Pressure response of the THz spectrum of bulk liquid water revealed by intermolecular instantaneous normal mode analysis. J. Chem. Phys. 2019, 150, 084502.

(8) Allen, A. E. A.; Payne, M. C.; Cole, D. J. Harmonic force constants for molecular mechanics force fields via Hessian matrix projection. J. Chem. Theory Comput. 2018, 14, 274-281.

(9) Miller, W. H. The semiclassical initial value representation: A potentially practical way for adding quantum effects to classical molecular dynamics simulations. J. Phys. Chem. A 2001, 105, 29422955.

(10) Richardson, J. O. Ring-polymer instanton theory. Int. Rev. Phys. Chem. 2018, 37, 171-216.

(11) Ceotto, M. Vibration-assisted tunneling: a semiclassical instanton approach. Mol. Phys. 2012, 110, 547-559.

(12) Gaigeot, M.-P. Some opinions on MD-based vibrational spectroscopy of gas phase molecules and their assembly: an overview 
of what has been achieved and where to go. Spectrochim. Acta, Part A 2021, 260, 119864.

(13) Wu, H.; Rahman, M.; Wang, J.; Louderaj, U.; Hase, W. L.; Zhuang, Y. Higher-accuracy schemes for approximating the Hessian from electronic structure calculations in chemical dynamics simulations. J. Chem. Phys. 2010, 133, 074101.

(14) Zhuang, Y.; Siebert, M. R.; Hase, W. L.; Kay, K. G.; Ceotto, M. Evaluating the Accuracy of Hessian Approximations for Direct Dynamics Simulations. J. Chem. Theory Comput. 2012, 9, 54-64.

(15) Ceotto, M.; Zhuang, Y.; Hase, W. L. Accelerated direct semiclassical molecular dynamics using a compact finite difference Hessian scheme. J. Chem. Phys. 2013, 138, 054116.

(16) Conte, R.; Gabas, F.; Botti, G.; Zhuang, Y.; Ceotto, M. Semiclassical vibrational spectroscopy with Hessian databases. J. Chem. Phys. 2019, 150, 244118.

(17) Broyden, C. G. A class of methods for solving nonlinear simultaneous equations. Math. Comput. 1965, 19, 577.

(18) Powell, M. J. D. Recent advances in unconstrained optimization. Math. Program. 1971, 1, 26-57.

(19) Dennis, J. E., Jr.; Moré, J. J. Quasi-Newton methods, motivation and theory. SIAM Rev. 1977, 19, 46-89.

(20) Nocedal, J. Theory of algorithms for unconstrained optimization. Acta Numer. 1992, 1, 199-242.

(21) Bofill, J. M. Updated Hessian matrix and the restricted step method for locating transition structures. J. Comput. Chem. 1994, 15, $1-11$.

(22) Denzel, A.; Kästner, J. Hessian matrix update scheme for transition state search based on Gaussian process regression. J. Chem. Theory Comput. 2020, 16, 5083-5089.

(23) Quinonero-Candela, J.; Rasmussen, C. E. A unifying view of sparse approximate Gaussian process regression. J. Mach. Learn. Res. 2005, 6, 1939-1959.

(24) Williams, C. K.; Rasmussen, C. E. Gaussian Processes for Machine Learning; MIT Press Cambridge: MA, 2006; Vol. 2.

(25) Deringer, V. L.; Bartók, A. P.; Bernstein, N.; Wilkins, D. M.; Ceriotti, M.; Csányi, G. Gaussian Process Regression for Materials and Molecules. Chem. Rev. 2021, 121, 10073-10141.

(26) Laude, G.; Calderini, D.; Tew, D. P.; Richardson, J. O. Ab initio instanton rate theory made efficient using Gaussian process regression. Faraday Discuss. 2018, 212, 237-258.

(27) Martinetz, T.; Schulten, K. A neural-gas network learns topologies. Artif. Neural Network 1991, 397-402.

(28) Martinetz, T. M.; Berkovich, S. G.; Schulten, K. J. "Neural-gas" network for vector quantization and its application to time-series prediction. IEEE Trans. Neural Network. 1993, 4, 558-569.

(29) Fritzke, B. A growing neural gas network learns topologies. Adv. Neural Inf. Process. Syst. 1995, 7, 625-632.

(30) Martinetz, T.; Schulten, K. Topology representing networks. Neural Network. 1994, 7, 507-522.

(31) Prudent, Y.; Ennaji, A. An incremental growing neural gas learns topologies. Proceedings 2005 IEEE International Joint Conference on Neural Networks, 2005; pp 1211-1216.

(32) Marsland, S.; Shapiro, J.; Nehmzow, U. A self-organising network that grows when required. Neural Network. 2002, 15, 10411058.

(33) Cottrell, M.; Hammer, B.; Hasenfuß, A.; Villmann, T. Batch and median neural gas. Neural Network. 2006, 19, 762-771.

(34) Tropsha, A. Best practices for QSAR model development, validation, and exploitation. Mol. Inf. 2010, 29, 476-488.

(35) Todeschini, R.; Consonni, V. Handbook of Molecular Descriptors; John Wiley \& Sons, 2008; Vol. 11.

(36) Cherkasov, A.; Muratov, E. N.; Fourches, D.; Varnek, A.; Baskin, I. I.; Cronin, M.; Dearden, J.; Gramatica, P.; Martin, Y. C.; Todeschini, R.; et al. QSAR modeling: where have you been? Where are you going to? J. Med. Chem. 2014, 57, 4977-5010.

(37) Ekins, S.; Puhl, A. C.; Zorn, K. M.; Lane, T. R.; Russo, D. P.; Klein, J. J.; Hickey, A. J.; Clark, A. M. Exploiting machine learning for end-to-end drug discovery and development. Nat. Mater. 2019, 18, 435-441.
(38) Dara, S.; Dhamercherla, S.; Jadav, S. S.; Babu, C.; Ahsan, M. J. Machine Learning in Drug Discovery: A Review. Artif. Intell. Rev. 2021, 1-53.

(39) Von Lilienfeld, O. A. Quantum machine learning in chemical compound space. Angew. Chem., Int. Ed. 2018, 57, 4164-4169.

(40) Botu, V.; Ramprasad, R. Adaptive machine learning framework to accelerate $\mathrm{ab}$ initio molecular dynamics. Int. J. Quantum Chem. 2015, 115, 1074-1083.

(41) Ye, S.; Hu, W.; Li, X.; Zhang, J.; Zhong, K.; Zhang, G.; Luo, Y.; Mukamel, S.; Jiang, J. A neural network protocol for electronic excitations of N-methylacetamide. Proc. Natl. Acad. Sci. U.S.A. 2019, $116,11612-11617$.

(42) Gastegger, M.; Behler, J.; Marquetand, P. Machine learning molecular dynamics for the simulation of infrared spectra. Chem. Sci. 2017, 8, 6924-6935.

(43) De, S.; Bartók, A. P.; Csányi, G.; Ceriotti, M. Comparing molecules and solids across structural and alchemical space. Phys. Chem. Chem. Phys. 2016, 18, 13754-13769.

(44) Moosavi, S. M.; Nandy, A.; Jablonka, K. M.; Ongari, D.; Janet, J. P.; Boyd, P. G.; Lee, Y.; Smit, B.; Kulik, H. J. Understanding the diversity of the metal-organic framework ecosystem. Nat. Commun. 2020, 11, 4068 .

(45) Gómez-Bombarelli, R.; Wei, J. N.; Duvenaud, D.; HernándezLobato, J. M.; Sánchez-Lengeling, B.; Sheberla, D.; AguileraIparraguirre, J.; Hirzel, T. D.; Adams, R. P.; Aspuru-Guzik, A. Automatic chemical design using a data-driven continuous representation of molecules. ACS Cent. Sci. 2018, 4, 268-276.

(46) Conte, R.; Ceotto, M. Quantum Chemistry and Dynamics of Excited States: Methods and Applications; John Wiley \& Sons, 2020; p 595.

(47) Ceotto, M.; Di Liberto, G.; Conte, R. Semiclassical "Divideand-Conquer" Method for Spectroscopic Calculations of High Dimensional Molecular Systems. Phys. Rev. Lett. 2017, 119, 010401.

(48) Lele, S. K. Compact finite difference schemes with spectral-like resolution. J. Comput. Phys. 1992, 103, 16-42.

(49) Lynch, R. E.; Rice, J. R. High accuracy finite difference approximation to solutions of elliptic partial differential equations. Proc. Natl. Acad. Sci. U.S.A. 1978, 75, 2541-2544.

(50) Zhuang, Y.; Sun, X.-H. A high order ADI method for separable generalized Helmholtz equations. Adv. Eng. Software 2000, 31, 585591.

(51) Zhuang, Y.; Sun, X.-H. A high-order fast direct solver for singular Poisson equations. J. Comput. Phys. 2001, 171, 79-94.

(52) Karandashev, K.; Vanícek, J. A combined on-the-fly/ interpolation procedure for evaluating energy values needed in molecular simulations. J. Chem. Phys. 2019, 151, 174116.

(53) Heller, E. J. The semiclassical way to molecular spectroscopy. Acc. Chem. Res. 1981, 14, 368-375.

(54) Golubev, N. V.; Begušić, T.; Vanícek, J. On-the-fly ab initio semiclassical evaluation of electronic coherences in polyatomic molecules reveals a simple mechanism of decoherence. Phys. Rev. Lett. 2020, 125, 083001.

(55) Begušić, T.; Vaníček, J. On-the-fly ab initio semiclassical evaluation of third-order response functions for two-dimensional electronic spectroscopy. J. Chem. Phys. 2020, 153, 184110.

(56) Kaledin, A. L.; Miller, W. H. Time averaging the semiclassical initial value representation for the calculation of vibrational energy levels. J. Chem. Phys. 2003, 118, 7174-7182.

(57) Kaledin, A. L.; Miller, W. H. Time averaging the semiclassical initial value representation for the calculation of vibrational energy levels. II. Application to $\mathrm{H} 2 \mathrm{CO}, \mathrm{NH} 3, \mathrm{CH} 4, \mathrm{CH} 2 \mathrm{D} 2$. J. Chem. Phys. 2003, 119, 3078-3084.

(58) Miller, W. H. Uniform semiclassical approximations for elastic scattering and eigenvalue problems. J. Chem. Phys. 1968, 48, 464467.

(59) Miller, W. H. Semiclassical nature of atomic and molecular collisions. Acc. Chem. Res. 1971, 4, 161-167. 
(60) Miller, W. H. Spiers memorial lecture quantum and semiclassical theory of chemical reaction rates. Faraday Discuss. 1998, 110, 1-21.

(61) Miller, W. H. Quantum dynamics of complex molecular systems. Proc. Natl. Acad. Sci. U.S.A. 2005, 102, 6660-6664.

(62) Sun, X.; Wang, H.; Miller, W. H. On the semiclassical description of quantum coherence in thermal rate constants. J. Chem. Phys. 1998, 109, 4190-4200.

(63) Thoss, M.; Wang, H.; Miller, W. H. Generalized forwardbackward initial value representation for the calculation of correlation functions in complex systems. J. Chem. Phys. 2001, 114, 9220-9235.

(64) Yamamoto, T.; Miller, W. H. Semiclassical calculation of thermal rate constants in full Cartesian space: The benchmark reaction $\mathrm{D}+\mathrm{H} 2 \rightarrow \mathrm{DH}+\mathrm{H}$. J. Chem. Phys. 2003, 118, 2135-2152.

(65) Heller, E. J. Frozen Gaussians: A very simple semiclassical approximation. J. Chem. Phys. 1981, 75, 2923-2931.

(66) Herman, M. F.; Kluk, E. A semiclasical justification for the use of non-spreading wavepackets in dynamics calculations. Chem. Phys. 1984, 91, 27-34.

(67) Miller, W. H. An alternate derivation of the Herman-Kluk (coherent state) semiclassical initial value representation of the time evolution operator. Mol. Phys. 2002, 100, 397-400.

(68) Kluk, E.; Herman, M. F.; Davis, H. L. Comparison of the propagation of semiclassical frozen Gaussian wave functions with quantum propagation for a highly excited anharmonic oscillator. J. Chem. Phys. 1986, 84, 326-334.

(69) Kay, K. G. Semiclassical propagation for multidimensional systems by an initial value method. J. Chem. Phys. 1994, 101, 22502260.

(70) Kay, K. G. Integral expressions for the semiclassical timedependent propagator. J. Chem. Phys. 1994, 100, 4377-4392.

(71) Kay, K. G. Numerical study of semiclassical initial value methods for dynamics. J. Chem. Phys. 1994, 100, 4432-4445.

(72) Kay, K. G. The Herman-Kluk approximation: Derivation and semiclassical corrections. Chem. Phys. 2006, 322, 3-12.

(73) Grossmann, F.; Xavier, A. L. From the coherent state path integral to a semiclassical initial value representation of the quantum mechanical propagator. Phys. Lett. A 1998, 243, 243-248.

(74) Antipov, S. V.; Ye, Z.; Ananth, N. Dynamically consistent method for mixed quantum-classical simulations: A semiclassical approach. J. Chem. Phys. 2015, 142, 184102.

(75) Church, M. S.; Antipov, S. V.; Ananth, N. Validating and implementing modified Filinov phase filtration in semiclassical dynamics. J. Chem. Phys. 2017, 146, 234104.

(76) Church, M. S.; Ananth, N. Semiclassical dynamics in the mixed quantum-classical limit. J. Chem. Phys. 2019, 151, 134109.

(77) Buchholz, M.; Fallacara, E.; Gottwald, F.; Ceotto, M.; Grossmann, F.; Ivanov, S. D. Herman-Kluk propagator is free from zero-point energy leakage. Chem. Phys. 2018, 515, 231-235.

(78) Heller, E. J. Cellular dynamics: A new semiclassical approach to time-dependent quantum mechanics. J. Chem. Phys. 1991, 94, 27232729.

(79) Di Liberto, G.; Ceotto, M. The Importance of the Preexponential Factor in Semiclassical Molecular Dynamics. J. Chem. Phys. 2016, 145, 144107.

(80) De Leon, N.; Heller, E. J. Semiclassical quantization and extraction of eigenfunctions using arbitrary trajectories. J. Chem. Phys. 1983, 78, 4005-4017.

(81) Ceotto, M.; Atahan, S.; Tantardini, G. F.; Aspuru-Guzik, A. Multiple coherent states for first-principles semiclassical initial value representation molecular dynamics. J. Chem. Phys. 2009, 130, 234113.

(82) Ceotto, M.; Atahan, S.; Shim, S.; Tantardini, G. F.; AspuruGuzik, A. First-principles semiclassical initial value representation molecular dynamics. Phys. Chem. Chem. Phys. 2009, 11, 3861-3867.

(83) Gabas, F.; Conte, R.; Ceotto, M. On-the-fly ab initio Semiclassical Calculation of Glycine Vibrational Spectrum. J. Chem. Theory Comput. 2017, 13, 2378.

(84) Ceotto, M.; Dell'Angelo, D.; Tantardini, G. F. Multiple coherent states semiclassical initial value representation spectra calculations of lateral interactions for $\mathrm{CO}$ on $\mathrm{Cu}(100)$. J. Chem. Phys. 2010, 133, 054701.

(85) Conte, R.; Aspuru-Guzik, A.; Ceotto, M. Reproducing Deep Tunneling Splittings, Resonances, and Quantum Frequencies in Vibrational Spectra From a Handful of Direct Ab Initio Semiclassical Trajectories. J. Phys. Chem. Lett. 2013, 4, 3407-3412.

(86) Micciarelli, M.; Gabas, F.; Conte, R.; Ceotto, M. An Effective Semiclassical Approach to IR Spectroscopy. J. Chem. Phys. 2019, 150, 184113.

(87) Micciarelli, M.; Conte, R.; Suarez, J.; Ceotto, M. Anharmonic vibrational eigenfunctions and infrared spectra from semiclassical molecular dynamics. J. Chem. Phys. 2018, 149, 064115.

(88) Tamascelli, D.; Dambrosio, F. S.; Conte, R.; Ceotto, M. Graphics processing units accelerated semiclassical initial value representation molecular dynamics. J. Chem. Phys. 2014, 140, 174109.

(89) Buchholz, M.; Grossmann, F.; Ceotto, M. Mixed semiclassical initial value representation time-averaging propagator for spectroscopic calculations. J. Chem. Phys. 2016, 144, 094102.

(90) Buchholz, M.; Grossmann, F.; Ceotto, M. Application of the mixed time-averaging semiclassical initial value representation method to complex molecular spectra. J. Chem. Phys. 2017, 147, 164110.

(91) Buchholz, M.; Grossmann, F.; Ceotto, M. Simplified approach to the mixed time-averaging semiclassical initial value representation for the calculation of dense vibrational spectra. J. Chem. Phys. 2018, $148,114107$.

(92) Ma, X.; Di Liberto, G.; Conte, R.; Hase, W. L.; Ceotto, M. A quantum mechanical insight into SN2 reactions: Semiclassical initial value representation calculations of vibrational features of the $\mathrm{Cl}-\cdots$ $\mathrm{CH} 3 \mathrm{Cl}$ pre-reaction complex with the VENUS suite of codes. J. Chem. Phys. 2018, 149, 164113.

(93) Conte, R.; Parma, L.; Aieta, C.; Rognoni, A.; Ceotto, M. Improved semiclassical dynamics through adiabatic switching trajectory sampling. J. Chem. Phys. 2019, 151, 214107.

(94) Aieta, C.; Micciarelli, M.; Bertaina, G.; Ceotto, M. Anharmonic quantum nuclear densities from full dimensional vibrational eigenfunctions with application to protonated glycine. Nat. Commun. 2020, 11, 4348 .

(95) Aieta, C.; Bertaina, G.; Micciarelli, M.; Ceotto, M. Representing molecular ground and excited vibrational eigenstates with nuclear densities obtained from semiclassical initial value representation molecular dynamics. J. Chem. Phys. 2020, 153, 214117.

(96) Ceotto, M.; Tantardini, G. F.; Aspuru-Guzik, A. Fighting the curse of dimensionality in first-principles semiclassical calculations: Non-local reference states for large number of dimensions. J. Chem. Phys. 2011, 135, 214108.

(97) Di Liberto, G.; Conte, R.; Ceotto, M. "Divide-and-conquer" semiclassical molecular dynamics: An application to water clusters. J. Chem. Phys. 2018, 148, 104302.

(98) Bertaina, G.; Di Liberto, G.; Ceotto, M. Reduced rovibrational coupling Cartesian dynamics for semiclassical calculations: Application to the spectrum of the Zundel cation. J. Chem. Phys. 2019, 151, 114307.

(99) Gabas, F.; Di Liberto, G.; Conte, R.; Ceotto, M. Protonated glycine supramolecular systems: the need for quantum dynamics. Chem. Sci. 2018, 9, 7894-7901.

(100) Gabas, F.; Di Liberto, G.; Ceotto, M. Vibrational investigation of nucleobases by means of divide and conquer semiclassical dynamics. J. Chem. Phys. 2019, 150, 224107.

(101) Gabas, F.; Conte, R.; Ceotto, M. Semiclassical vibrational spectroscopy of biological molecules using force fields. J. Chem. Theory Comput. 2020, 16, 3476-3485.

(102) Rognoni, A.; Conte, R.; Ceotto, M. How many water molecules are needed to solvate one? Chem. Sci. 2021, 12, 20602064.

(103) Rognoni, A.; Conte, R.; Ceotto, M. Caldeira-Leggett model vs $\mathrm{ab}$ initio potential: A vibrational spectroscopy test of water solvation. J. Chem. Phys. 2021, 154, 094106.

(104) Cazzaniga, M.; Micciarelli, M.; Moriggi, F.; Mahmoud, A.; Gabas, F.; Ceotto, M. Anharmonic calculations of vibrational spectra 
for molecular adsorbates: A divide-and-conquer semiclassical molecular dynamics approach. J. Chem. Phys. 2020, 152, 104104.

(105) Gandolfi, M.; Rognoni, A.; Aieta, C.; Conte, R.; Ceotto, M. Machine learning for vibrational spectroscopy via divide-and-conquer semiclassical initial value representation molecular dynamics with application to N-methylacetamide. J. Chem. Phys. 2020, 153, 204104. (106) Brewer, M. L.; Hulme, J. S.; Manolopoulos, D. E. Semiclassical dynamics in up to 15 coupled vibrational degrees of freedom. J. Chem. Phys. 1997, 106, 4832-4839.

(107) Bowman, J. M.; Wierzbicki, A.; Zúñiga, J. Exact vibrational energies of non-rotating $\mathrm{H} 2 \mathrm{O}$ and $\mathrm{D} 2 \mathrm{O}$ using an accurate $\mathrm{ab}$ initio potential. Chem. Phys. Lett. 1988, 150, 269-274.

(108) Martin, J. M. L.; Lee, T. J.; Taylor, P. R. An accurate ab initio quartic force field for formaldehyde and its isotopomers. J. Mol. Spectrosc. 1993, 160, 105-116.

(109) Lee, T. J.; Martin, J. M. L.; Taylor, P. R. An accurate ab initio quartic force field and vibrational frequencies for $\mathrm{CH} 4$ and isotopomers. J. Chem. Phys. 1995, 102, 254-261.

(110) Nandi, A.; Qu, C.; Bowman, J. M. Full and fragmented permutationally invariant polynomial potential energy surfaces for trans and cis $\mathrm{N}$-methyl acetamide and isomerization saddle points. J. Chem. Phys. 2019, 151, 084306.

(111) Conte, R.; Qu, C.; Houston, P. L.; Bowman, J. M. Efficient Generation of Permutationally Invariant Potential Energy Surfaces for Large Molecules. J. Chem. Theory Comput. 2020, 16, 3264-3272.

(112) Valiev, M.; Bylaska, E. J.; Govind, N.; Kowalski, K.; Straatsma, T. P.; Van Dam, H. J. J.; Wang, D.; Nieplocha, J.; Apra, E.; Windus, T. L.; de Jong, W. A. NWChem: A comprehensive and scalable opensource solution for large scale molecular simulations. Comput. Phys. Commun. 2010, 181, 1477-1489.

(113) Di Liberto, G.; Conte, R.; Ceotto, M. "Divide and conquer" semiclassical molecular dynamics: A practical method for spectroscopic calculations of high dimensional molecular systems. J. Chem. Phys. 2018, 148, 014307.

(114) Biswal, H. S.; Gloaguen, E.; Loquais, Y.; Tardivel, B.; Mons, M. Strength of NH..S Hydrogen Bonds in Methionine Residues Revealed by Gas-Phase IR/UV Spectroscopy. J. Phys. Chem. Lett. 2012, 3, 755-759.

(115) Begušić, T.; Cordova, M.; Vaníček, J. Single-Hessian thawed Gaussian approximation. J. Chem. Phys. 2019, 150, 154117. 